\title{
Cerebrospinal fluid concentrations of extracellular matrix proteins in
}

\section{Alzheimer's disease}

Karolina Minta ${ }^{1}$, Erik Portelius ${ }^{1,2}$, Shorena Janelidze ${ }^{3}$, Oskar Hansson ${ }^{3}$, Henrik

Zetterberg $^{1,2,4,5}$, Kaj Blennow ${ }^{1,2}$, Ulf Andreasson ${ }^{1,2}$

${ }^{1}$ Institute of Neuroscience and Physiology, Department of Psychiatry and

Neurochemistry, the Sahlgrenska Academy at the University of Gothenburg, Sweden

${ }^{2}$ Clinical Neurochemistry Laboratory, Sahlgrenska University Hospital, Mölndal,

Sweden ${ }^{3}$ Clinical Memory Research Unit, Department of Clinical Sciences, Lund

University, Sweden ${ }^{4}$ Department of Neurodegenerative Disease, UCL Institute of

Neurology, London, UK ${ }^{5}$ UK Dementia Research Institute at UCL, London, UK

Corresponding author:

Karolina Minta

Department of Psychiatry and Neurochemistry

Sahlgrenska University Hospital/Mölndal

S-431 80 Mölndal, Sweden

e-mail: karolina.minta@neuro.gu.se

tel: +46735660741 


\section{Abstract}

Background: Brevican, neurocan, tenascin- $\mathrm{C}$ and tenascin- $\mathrm{R}$ are extracellular matrix (ECM) proteins that are mainly expressed in the brain. They play important roles in proliferation, migration and differentiation of neurons and other cell types in the brain. They are also expressed in various pathological conditions, including reactive gliosis. The aim of the study was to investigate if ECM protein concentrations in cerebrospinal fluid (CSF) are linked to the neurodegenerative process in Alzheimer's disease $(A D)$.

Methods: Lumbar CSF samples from a non-AD control group $(n=50)$ and a clinically diagnosed $A D$ group $(n=42)$, matched for age and gender, were analyzed using commercially available ELISAs detecting ECM proteins. Mann-Whitney U test were used to examine group differences, while Spearman's rho test was used for correlations.

Results: Brevican, neurocan and tenascin-R concentrations in AD patients did not differ compared to healthy controls.

Conclusions: ECM proteins do not reflect AD-pathology in CSF.

Keywords: Alzheimer's disease, brevican; neurocan; tenascin-C; tenascin-R.

List of abbreviations: Alzheimer's disease $(A D)$, amyloid beta $(A B)$, central nervous system (CSN), cerebrospinal fluid (CSF), chondroitin sulphate proteoglycans (CSPG), coefficient of variation (CV), extracellular matrix (ECM), neurofibrillary tangles (NFTs), phosporylated tau (p-tau), senile plaques (SPs), total tau (t-tau). 



\section{Introduction}

Alzheimer's disease (AD) is an irreversible and progressive brain disorder, and the most common form of dementia. The risk of $A D$ is clearly correlated with increasing age and, clinically, the disease is characterized by memory loss and other forms of cognitive impairment (1). Almost 50 million people worldwide are affected by AD and this number is growing (2). Treatments for AD symptoms are available (3), however there is still no approved disease-modifying drug. Major pathological hallmarks of AD include extracellular senile plaques (SPs) formed by amyloid $\beta(A \beta)$ fibrils and intracellular neurofibrillary tangles (NFTs) formed by hyperphosporylated tau protein (1). There is a worldwide effort to find early biomarkers for the disease, which detect pathological changes before symptoms are noticeable. There are three protein biomarkers in cerebrospinal fluid (CSF), which can be used to detect AD pathologies: total tau (t-tau), phosphorylated tau (p-tau) and the 42 amino acid form of $A \beta$ (4). These biomarkers are now part of the research diagnostic criteria for $\operatorname{AD}(5,6)$ but there is still a need for new biomarkers that can be used for example in differential diagnostics and for prognosis.

Extracellular matrix $(E C M)$ components are believed to contribute to $A D$ progression. The function of ECM is to provide physical support to surrounding cells as well as to regulate intercellular communication (7); its major components are proteoglycans and glycoproteins. They play important role in cell adhesion, migration, neurite outgrowth and guidance (8). Brevican and neurocan are nervous-specific chondroitin sulphate proteoglycans (CSPG) (9), while tenascin- $C$ and tenascin- $R$ are large glycoproteins, where tenascin- $C$ is expressed in connective tissues (10), whereas tenascin- $R$ is abundant in nervous tissue (9). Brevican, neurocan and both tenascins are expressed by neuronal and glial cells $(9,11-13)$. As a response to the production of 
cytokines by activated microglia, astrocytes secrete proteoglycans around the areas of CNS tissue damage, leading to the formation of glial scars. This structure is not only a barrier to axonal regeneration, but it also inhibits the spread of the damage to other areas (14). Glial scars mainly consist of CSPGs and tenascin-R (15). Tenascin$C$ is also upregulated in inflammation (16) and upon microglial activation in $A D$ (ref), where $A \beta$ plaques and neurofibrillary tangles are surrounded by reactive astrocytes (14).

The aim of the study was to investigate if there are any alterations in the concentrations of these ECM proteins in CSF from AD patients compared to healthy controls.

\section{Materials and methods}

The study was conducted according to the Helsinki Declaration and approved by the regional ethical board in Lund (\#2016/1053).

The study involved 42 AD patients and 50 healthy controls (Table 1). AD patients had abnormal core AD CSF biomarker (Aß42, t-tau and p-tau) levels, while controls had normal levels. One AD patient lacked CSF AD biomarker results. The two groups were age- and gender-matched. CSF samples were collected by lumbar puncture procedures. Mini Mental State Examination (MMSE) was used to measure cognitive impairment.

Brevican, neurocan, tenascin- $\mathrm{C}$ and tenascin-R concentrations in CSF were measured using sandwich ELISA (RayBiotech, Norcross, GA, USA). The dilution factors were: 1:400 for brevican, 1:10 for neurocan and 1:2 for both tenascins. Quality control CSF pools were run at the beginning and the end of each assay to evaluate possible inter and intra alteration. The coefficients of variation (CVs) for the repeatability were below 
$11 \%$ and $\mathrm{CV}$ s for the intermediate precision measurements were below $19 \%$. The CSF samples were measured in singlicates due to volume limitations.

Because most of the CSF results were non-normally distributed, group comparisons were made using the Mann-Whitney $U$ test. Associations between variables were reported by Spearman's rank correlation coefficient. The analyses were performed using SPSS software, version 25 or GraphPad Prism, version 7. All tests were twosided and statistical significance was defined as $p \leq 0.05$.

\section{Results}

Patient demographics together with biomarker concentrations are shown in Table 1. Brevican, neurocan, tenascin- $\mathrm{C}$ and tenascin- $\mathrm{R}$ concentrations did not differ between $A D$ and control groups (Fig.1). Brevican, neurocan and tenascin- $R$ values correlated with each other in the control and AD groups separately as well as in the two groups combined (rho=0.68-0.77, $p<0.05$ ). Neurocan correlated with $A \beta 40$ ( $r h o=0.60$, $\mathrm{p}<0.05)$ in both groups, whilst its correlation with t-tau was only observed in the control group (rho $=0.63, p<0.05$ ). CSF ECM protein concentrations were similar in men and women in the control group, however tenascin- $\mathrm{C}$ and tenascin- $\mathrm{R}$ concentrations were significantly higher in women than in men in the AD group ( $p=0.02$ for both biomarkers, Fig.2).

\section{Discussion}

Previous studies using in house (non-commercial) assays for the ECM proteins and small samples were inconclusive (data not shown) and here results from larger and well-characterized AD-control cohorts is presented. The results indicate that CSF concentrations of brevican, neurocan, tenascin- $\mathrm{C}$ and tenascin- $\mathrm{R}$ are not changed in 
$A D$ even though several studies suggest that ECM proteins are involved in $A D$ pathophysiology. For example, CSPGs have been found in SPs (17) and they are known to inhibit the degradation of SPs and contribute to their persistence and even propagation in AD (8). CSPGs bind to fibrillar $A \beta$ and inhibit its proteolytic degradation (18). They inhibit the removal of $A \beta$ deposits by microglia (19) and they promote $A \beta$ aggregation into insoluble amyloid fibrils (20). It has also been shown that $A \beta$ aggregation alters the structure and proteolytic cleavage of brevican (21). Moreover, matrix metalloproteinases that cleave brevican lost their activity in the same model, consequently reducing the amount of an N-terminal proteolytic fragment of brevican (21). A $\beta$ accumulation was observed to upregulate neurocan expression (22). Tenascin- $C$ was found to form extracellular deposits around $A \beta$ plaques (23) and its transcription was upregulated in response to $A \beta$ exposure $(16,23)$. Estrogens are thought to play a role in gender difference observed in many neurological diseases, including AD (24). Previous studies reported that women are at increased risk of developing $\operatorname{AD}(24,25)$, which might be associated with a reduction of brain estrogen levels in postmenopausal women compared to agematched men (24). AD pathogenesis strongly interacts with immunological mechanisms in the brain and frequency of complications related to inflammatory conditions are greater in women $(26,27)$. Our observation of increased tenascin concentrations in women could potentially reflect these sex differences, although more studies are needed to draw any firm conclusions on this.

In conclusion, strong correlations between CSF concentrations of brevican, neurocan and tenascin-R in both $A D$ and control groups suggest that these ECM proteins reflect the same neurophysiological processes occurring in the CNS, whilst the lack 
of $A D$ vs. control group differences speak against any strong association with the pathological process of AD.

Table1. Demographic and biomarkers 


\begin{tabular}{llll}
\hline Characteristic & & $\begin{array}{l}\text { AD } \\
(\mathrm{n}=42)\end{array}$ & $\begin{array}{l}\text { Controls } \\
(\mathrm{n}=50)\end{array}$ \\
\hline Gender, $\mathrm{n}(\%)$ & Male & $13(31 \%)$ & $16(32 \%)$ \\
& Female & $29(69 \%)$ & $34(68 \%)$ \\
Age, median (IQR) & $75(11.8)$ & $75(9.75)$ \\
Protein concentration, median (IQR) $[\mathrm{ng} / \mathrm{ml}]$ & \\
ECM proteins & Brevican & $466(250)$ & $521(216)$ \\
& Neurocan & $37.6(14.4)$ & $38.9(13.4)$ \\
& Tenascin-C & $0.118(0.0536)$ & $0.102(0.0638)$ \\
& Tenascin-R & $1.78(0.828)$ & $1.82(0.748)$ \\
AD biomarkers & A 40 & $6.29(2.50)$ & $6.33(2.56)$ \\
& Aß42 & $0.411(0.156)$ & $0.789(0.461)$ \\
& T-tau & $0.606(0.247)$ & $0.319(0.131)$ \\
& P-tau & $0.106(0.0571)$ & $0.0431(0.0205)$ \\
\hline & & & \\
\hline
\end{tabular}


A

BREVICAN

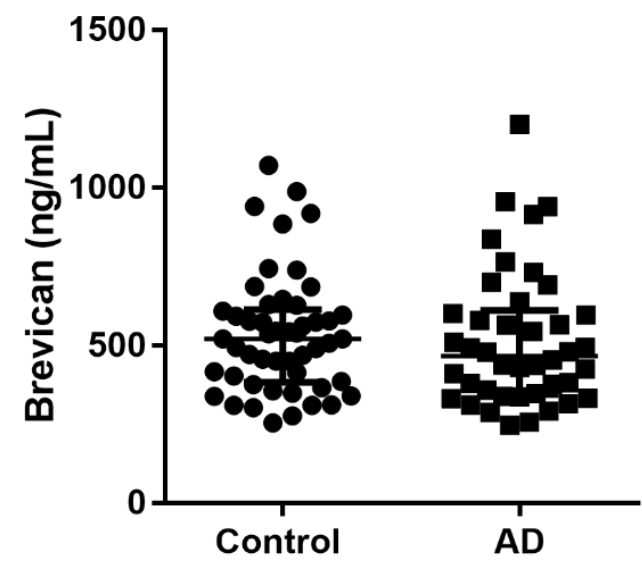

C
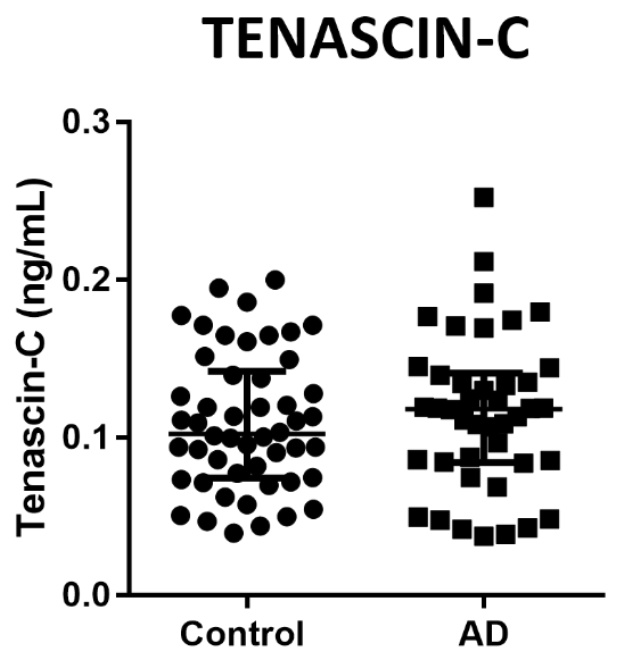

B NEUROCAN

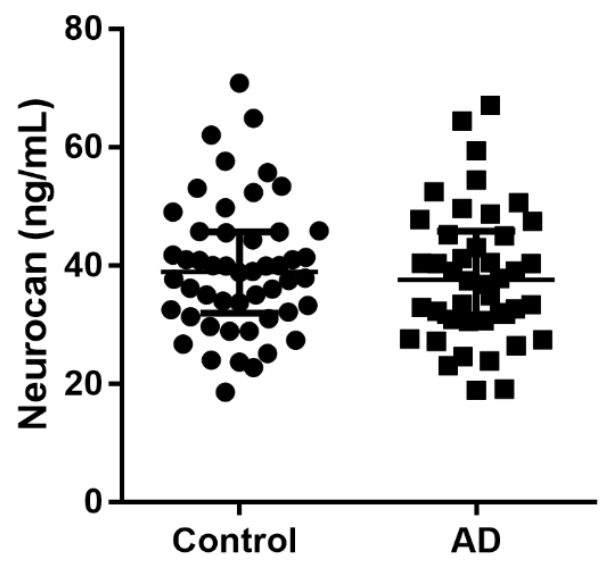

D TENASCIN-R

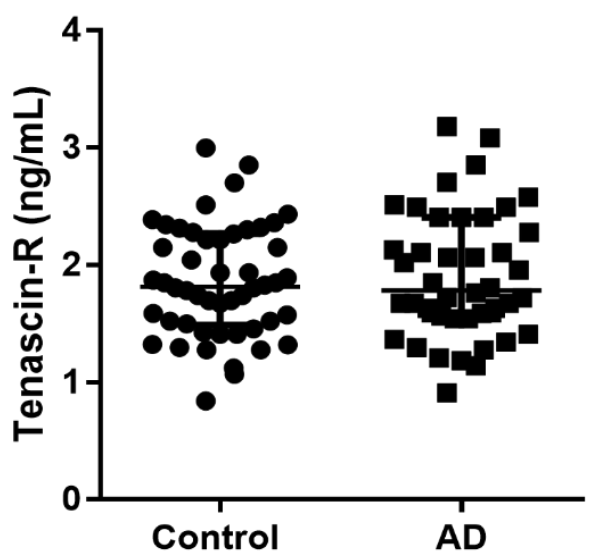

Fig.1 ECM proteins in CSF from AD patients compared to healthy controls.

Represented as median with interquartile range. 
A

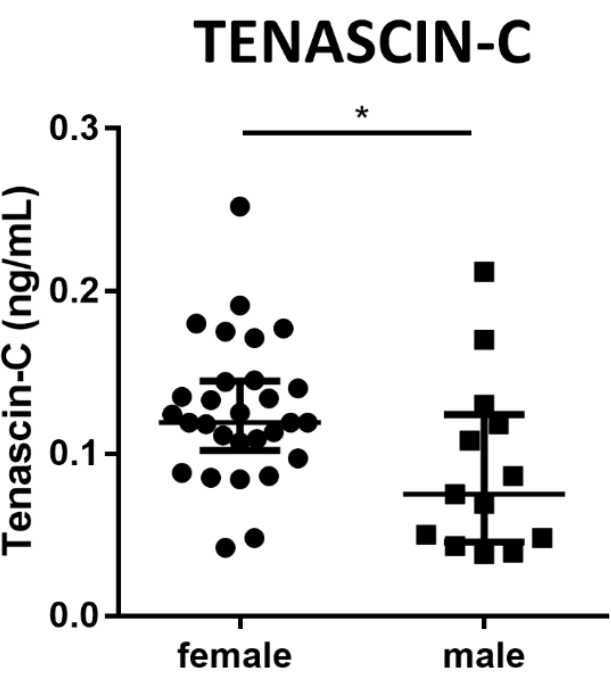

B TENASCIN-R



Fig.2 Tenascin-C (A) and tenascin- $\mathrm{R}(\mathrm{B})$ protein concentrations in female and male groups of $A D$ patients.

Represented as median with interquartile range.

Significance: ${ }^{*}=p \leq 0.05$ 
1. Blennow K, de Leon MJ, Zetterberg H. Alzheimer's disease. Lancet. 2006;368(9533):387-403.

2. Prince M, Bryce R, Albanese E, Wimo A, Ribeiro W, Ferri CP. The global prevalence of dementia: a systematic review and metaanalysis. Alzheimers Dement. 2013;9(1):63-75 e2.

3. Yiannopoulou KG, Papageorgiou SG. Current and future treatments for Alzheimer's disease. Ther Adv Neurol Disord. 2013;6(1):19-33.

4. Blennow K, Hampel H, Weiner M, Zetterberg H. Cerebrospinal fluid and plasma biomarkers in Alzheimer disease. Nat Rev Neurol. 2010;6(3):131-44.

5. Jack CR, Jr., Bennett DA, Blennow K, Carrillo MC, Dunn B, Haeberlein SB, et al. NIA-AA Research Framework: Toward a biological definition of Alzheimer's disease. Alzheimers Dement. 2018;14(4):535-62.

6. Dubois B, Feldman $\mathrm{HH}$, Jacova $\mathrm{C}$, Hampel $\mathrm{H}$, Molinuevo JL, Blennow K, et al. Advancing research diagnostic criteria for Alzheimer's disease: the IWG-2 criteria. Lancet Neurol. 2014;13(6):614-29.

7. Mouw JK, Ou G, Weaver VM. Extracellular matrix assembly: a multiscale deconstruction. Nat Rev Mol Cell Biol. 2014;15(12):771-85.

8. Cui H, Freeman C, Jacobson GA, Small DH. Proteoglycans in the central nervous system: role in development, neural repair, and Alzheimer's disease. IUBMB Life. 2013;65(2):108-20.

9. Yamaguchi Y. Lecticans: organizers of the brain extracellular matrix. Cell Mol Life Sci. 2000;57(2):276-89.

10. Lowy CM, Oskarsson T. Tenascin C in metastasis: A view from the invasive front. Cell Adh Migr. 2015;9(1-2):112-24. 
11. Frischknecht R, Seidenbecher Cl. Brevican: a key proteoglycan in the perisynaptic extracellular matrix of the brain. Int J Biochem Cell Biol. 2012;44(7):1051-4.

12. Ferhat L, Chevassus-Au-Louis N, Khrestchatisky M, Ben-Ari Y, Represa A. Seizures induce tenascin-C mRNA expression in neurons. J Neurocytol. 1996;25(9):535-46.

13. Becker CG, Becker T, Meyer RL, Schachner M. Tenascin-R inhibits the growth of optic fibers in vitro but is rapidly eliminated during nerve regeneration in the salamander Pleurodeles waltl. J Neurosci. 1999;19(2):813-27.

14. Siebert JR, Conta Steencken A, Osterhout DJ. Chondroitin sulfate proteoglycans in the nervous system: inhibitors to repair. Biomed Res Int. $2014 ; 2014: 845323$

15. Fournier AE, Strittmatter SM. Repulsive factors and axon regeneration in the CNS. Curr Opin Neurobiol. 2001;11(1):89-94.

16. Xie K, Liu Y, Hao W, Walter S, Penke B, Hartmann T, et al. Tenascin-C deficiency ameliorates Alzheimer's disease-related pathology in mice. Neurobiol Aging. 2013;34(10):2389-98.

17. DeWitt DA, Silver J, Canning DR, Perry G. Chondroitin sulfate proteoglycans are associated with the lesions of Alzheimer's disease. Exp Neurol. $1993 ; 121(2): 149-52$

18. Gupta-Bansal R, Frederickson RC, Brunden KR. Proteoglycan-mediated inhibition of $A$ beta proteolysis. A potential cause of senile plaque accumulation. $J$ Biol Chem. 1995;270(31):18666-71. 
19. Shaffer LM, Dority MD, Gupta-Bansal R, Frederickson RC, Younkin SG, Brunden KR. Amyloid beta protein (A beta) removal by neuroglial cells in culture. Neurobiol Aging. 1995;16(5):737-45.

20. Ariga T, Miyatake T, Yu RK. Role of proteoglycans and glycosaminoglycans in the pathogenesis of Alzheimer's disease and related disorders: amyloidogenesis and therapeutic strategies--a review. J Neurosci Res. 2010;88(11):2303-15.

21. Ajmo JM, Bailey LA, Howell MD, Cortez LK, Pennypacker KR, Mehta $\mathrm{HN}$, et al. Abnormal post-translational and extracellular processing of brevican in plaque-bearing mice over-expressing APPsw. J Neurochem. 2010;113(3):784-95. 22. Yan H, Zhu X, Xie J, Zhao Y, Liu X. beta-amyloid increases neurocan expression through regulating Sox9 in astrocytes: A potential relationship between Sox9 and chondroitin sulfate proteoglycans in Alzheimer's disease. Brain Res. 2016;1646:377-83.

23. Mi Z, Halfter W, Abrahamson EE, Klunk WE, Mathis CA, Mufson EJ, et al. Tenascin-C Is Associated with Cored Amyloid-beta Plaques in Alzheimer Disease and Pathology Burdened Cognitively Normal Elderly. J Neuropathol Exp Neurol. 2016;75(9):868-76.

24. Li R, Cui J, Shen Y. Brain sex matters: estrogen in cognition and Alzheimer's disease. Mol Cell Endocrinol. 2014;389(1-2):13-21.

25. Patel M. Alzheimer disease: Revising the risk of Alzheimer disease in women. Nat Rev Neurol. 2017;13(10):575.

26. Whitacre CC. Sex differences in autoimmune disease. Nat Immunol. 2001;2(9):777-80. 
27. Lockshin MD. Sex differences in autoimmune disease. Lupus.

2006;15(11):753-6. 